\title{
DESENVOLVIMENTO DO COEFICIENTE PARCIAL DE ERGONOMIA E SEGURANÇA EM TRATORES AGRÍCOLAS ${ }^{1}$
}

\author{
HENRIQUE DEBIASI ${ }^{2}$, JOSÉ F. SCHLOSSER ${ }^{3}$, EDER D. PINHEIRO ${ }^{4}$
}

RESUMO: O presente trabalho teve como objetivo criar e aplicar o coeficiente parcial de ergonomia e segurança em tratores agrícolas (COPES). O COPES corresponde a um valor numérico variável de 0 a 100, que expressa a qualidade em termos de ergonomia e segurança dos tratores agrícolas. $\mathrm{O}$ referido coeficiente foi desenvolvido e avaliado por meio da verificação da presença e estado de conservação de itens relacionados à ergonomia e segurança, em 175 tratores agrícolas usados, presentes em 114 propriedades amostradas junto às microrregiões gaúchas de Santa Maria, Restinga Seca e Cachoeira do Sul. Os resultados mostraram que o COPES apresentou grande variação entre os tratores amostrados $(7,64$ a 84,80$)$, sendo a média geral inferior a 35 . Isso demonstra que os tratores agrícolas, atualmente em uso na região abrangida por esta pesquisa, se caracterizam por más condições relacionadas à ergonomia e segurança. Além disso, o valor do COPES mostrou que diminui em escala logarítmica conforme aumenta o tempo de uso dos tratores $\left(\mathrm{r}^{2}=0,8018\right)$. Assim, pode-se concluir que o COPES é uma importante ferramenta para a avaliação quantitativa das condições de ergonomia e segurança em tratores agrícolas, demonstrando sensibilidade às variações apresentadas no que se refere a essas características.

PALAVRAS-CHAVE: mecanização agrícola, ergonomia, segurança.

\section{DEVELOPMENT OF PARTIAL ERGONOMIC AND SAFETY COEFFICIENT OF AGRICULTURAL TRACTORS}

SUMMARY: The objective of this work was to create and apply the partial ergonomic and safety coefficient of agricultural tractors (COPES). The COPES is a numeric value variable from 0 to 100 , showing ergonomic and safety conditions of agricultural tractors. This coefficient was developed and tested through checking the presence and conservation condition of ergonomic and safety items in 175 used agricultural tractors belonging to 114 farms localized in the regions of Santa Maria, Restinga Seca and Cachoeira do Sul, Brazil. The results showed that the COPES presented a considerable variation between the agricultural tractors sampled (7.64 to 84.80). The COPES mean value was inferior to 35. This indicates the unsatisfactory ergonomic and safety conditions of the agricultural tractors used in these regions. The COPES value tends to decrease in logarithm scale according to the agricultural tractors age $\left(\mathrm{r}^{2}=0.8018\right)$. Therefore, it is possible to conclude that the COPES is a relevant tool to evaluate numerically ergonomic and safety conditions in agricultural tractors, demonstrating sensibility to the variations presented by the tractors regarding to these characteristics.

KEYWORDS: agricultural mechanization, ergonomics, safety.

\footnotetext{
${ }^{1}$ Extraído da dissertação de mestrado do primeiro autor.

${ }^{2}$ Eng ${ }^{\mathrm{o}}$ Agrícola, M.Sc., Pesquisador, Centro de Pesquisa em Forrageiras, FEPAGRO, BR 290, km 412, São Gabriel - RS, Fone: (0XX55) 232.5411, e-mail: henriquedebiasi@yahoo.com.br.

${ }^{3}$ Eng $^{\text {O }}$ Agrônomo, Professor Titular, Departamento de Engenharia Rural, Centro de Ciências Rurais, UFSM, Santa Maria - RS.

${ }^{4}$ Acadêmico do Curso de Agronomia, UFSM, Monitor de Máquinas Agrícolas.

Recebido pelo Conselho Editorial em: 13-5-2003

Aprovado pelo Conselho Editorial em: 26-7-2004
} 


\section{INTRODUÇÃO}

Os acidentes de trabalho são o resultado de atos e condições inseguras (ZÓCCHIO, 1971; CUTULI et al., 1977; SCHLOSSER \& DEBIASI, 2001). Considerando a operação de tratores agrícolas, as condições inseguras referem-se à máquina (trator + implemento) e ao ambiente onde o trabalho está sendo realizado (condições do terreno, por exemplo). Nesse sentido, pesquisas, como as realizadas por SUUTARINEN (1991), ERIKSON (1996) e SCHLOSSER \& DEBIASI (2001), têm mostrado que os tratores agrícolas são os que mais colaboram para a geração de condições inseguras durante o trabalho com esse tipo de máquina.

Embora as estatísticas apontem que apenas $20 \%$ dos acidentes de trabalho, tanto na indústria quanto na agricultura, são ocasionados por condições inseguras (CUTULI et al., 1977; MÁRQUEZ, 1990; SCHLOSSER \& DEBIASI, 2001), esse fator não pode ser negligenciado. Além de se constituir em causa direta de acidentes de trabalho, um trator agrícola ergonomicamente mal projetado aumenta o nível de estresse físico e mental ao qual o operador se encontra submetido, levando esse a cometer erros (atos inseguros) que podem resultar em acidentes (WITNEY, 1988; MÁRQUEZ, 1990; PURCELL, 1996; YADAV \& TEWARI, 1998). Por outro lado, uma vez processado o acidente, seja por atos, seja por condições inseguras, os danos físicos ao operador podem ser minimizados ou mesmo eliminados se o trator apresentar dispositivos de segurança que, conforme CARDELLA (1999), visem a proteger as pessoas por meio da eliminação, isolamento e/ou sinalização daqueles componentes que podem causar lesões.

Quando se processa a avaliação dos tratores agrícolas com vistas à prevenção de acidentes, é importante considerar, além da presença dos itens relacionados à ergonomia e segurança, o estado de conservação dos mesmos. Dados obtidos por FEBO \& PESSINA (1995), por meio da avaliação de 96 tratores presentes na região Norte da Itália, mostraram que os agricultores daquele país tendem a negligenciar a manutenção de itens de ergonomia e segurança, em função de os mesmos não serem imprescindíveis ao uso do trator como fonte de potência.

Percebe-se, assim, que a qualidade ergonômica e a segurança de um determinado trator agrícola envolvem a avaliação da presença, bem como do estado de conservação de um grande número de itens, dificultando uma avaliação geral da máquina sob esse aspecto. Essa dificuldade pode ser sanada por meio da criação de uma medida quantitativa para diferenciar os tratores agrícolas em termos de sua qualidade em ergonomia e segurança. Assim, o objetivo deste trabalho foi criar e aplicar a campo a metodologia de cálculo do coeficiente parcial de ergonomia e segurança em tratores agrícolas (COPES), visando a disponibilizar uma ferramenta que permita uma avaliação de forma quantitativa e comparável da qualidade dos tratores agrícolas em relação à ergonomia e segurança.

\section{MATERIAL E MÉTODOS}

A pesquisa foi realizada nas microrregiões gaúchas de Santa Maria, Restinga Seca e Cachoeira do Sul. No total, foram avaliados 175 tratores agrícolas de várias marcas, modelos e anos de fabricação, pertencentes a 114 propriedades. Para o cálculo do COPES, os itens avaliados nos tratores agrícolas foram agrupados em três classes: características de segurança, ergonomia e gerais. Essas classes deram origem, respectivamente, ao coeficiente parcial de segurança (CPS), ao coeficiente parcial de ergonomia (CPE) e ao coeficiente parcial relativo às condições gerais (CPCG). O COPES, assim como cada um dos coeficientes que o compõem, pode assumir qualquer valor entre 0 e 100 . Para o cálculo dos referidos coeficientes, foi atribuída uma pontuação de 0 a 10 para cada um dos itens que o compunham, conforme a existência ou não do dispositivo, bem como em função do seu tipo.

Os itens e critérios utilizados para o cálculo do CPS foram os seguintes: 
- Proteção das pás do ventilador; invólucro adicional para isolamento do eixo da TDP; advertência sobre o risco de tombamento; advertência sobre o risco na TDP; orientações sobre o acoplamento de implementos; orientações sobre a partida no motor; existência de degraus de acesso; adequação da altura do primeiro degrau em relação ao solo à NBR ISO 4254-1 (1999); adequação da largura dos degraus à NBR ISO 4254-1 (1999); adequação da distância entre os degraus à NBR ISO 4254-1 (1999); superfície antiderrapante nos degraus; batente vertical nos degraus de acesso; inclinação dos degraus; dispositivo de drenagem de água no posto de operação; superfície antiderrapante na plataforma de operação; superfície antiderrapante nos pedais; dispositivo contra partida acidental; proteção da tubulação de escape; desligamento rápido da TDP; coloração vermelha do comando de acionamento/desligamento da TDP; possibilidade de controle do sistema hidráulico de levante de três pontos fora do posto de operação; dispositivo que prende os pedais do freio; acelerador de pé; cinto de segurança; luz de freio; luz de ré; indicadores de direção; luzes de advertência; faróis dianteiros; lanternas traseiras; farol de trabalho; encosto do assento; buzina; espelho retrovisor; contrapesos dianteiros; assento adicional; sistema hidráulico de levante de três pontos com dispositivo de engate rápido: Não (0 ponto); Sim (10 pontos) - pontuação em separado para cada um dos itens citados;

- Presença de estrutura de proteção contra o capotamento: ausente ( 0 ponto); tipo arco de segurança (9 pontos); estrutura de proteção de quatro pontos (10 pontos);

- Proteção da TDP: ausente (0 ponto); tipo tampa/escudo (7 pontos); tipo invólucro (10 pontos);

- Corrimões e/ou manípulos para o acesso e a saída do posto de operação: não (0 ponto); um (5 pontos); dois (10 pontos);

- Tipo de posto de operação: acavalado (0 ponto); plataforma (10 pontos);

- Condições dos pneus: avaliada em função da porcentagem da altura da garra dos pneus traseiros medida em relação à altura de garra original (pneu novo), sendo essa considerada como sendo $33 \mathrm{~mm}$ para os pneus do tipo R1 (coxilha) e de $75 \mathrm{~mm}$ para os do tipo R2 (arrozeiros). Os valores foram atribuídos segundo os seguintes critérios: menos de $25 \%$ (2,5 pontos); $25-50 \%$ (5 pontos); 50 $75 \%$ (7,5 pontos); e 75-100\% (10 pontos). Com a presença de rasgos e/ou rachaduras, foi reduzido 2,5 pontos no valor atribuído, e

- Segurança dos comandos: a pontuação em relação a esse item foi atribuída levando-se em consideração a existência ou não de comandos com superfícies pontiagudas, por meio da seguinte equação:

$$
\mathrm{P}_{\mathrm{i}}=10-((10 / \mathrm{n}) \mathrm{k})
$$

em que,

$\mathrm{P}_{\mathrm{i}}$ - pontuação referente a este item;

$\mathrm{n}$ - número total de comandos avaliados, e

$\mathrm{k}$ - corresponde ao número de comandos que possuem superfície pontiaguda.

Depois disso, foi efetuada a correção da pontuação daqueles itens em que o estado de conservação foi avaliado (Tabela 1), por meio da seguinte equação:

$$
\mathrm{P}_{\mathrm{c}}=\left(\mathrm{P}_{\mathrm{i}} \mathrm{y}\right) / 4
$$

em que,

$P_{c}$ - pontuação corrigida do item;

$\mathrm{P}_{\mathrm{i}}$ - pontuação inicial do item, e

y - nota obtida pelo item em razão de seu estado de conservação (valor entre 0 e 4 ). 
A avaliação do estado de conservação (variável "y" da eq.(2)) foi feita por meio da adaptação da metodologia proposta por FEBO \& PESSINA (1995). Nesse sentido, a cada um dos itens listados na Tabela 1, foi atribuída uma nota conforme o número de defeitos observados: 0 - dispositivo ausente ou retirado; 1 - dispositivo com três ou mais defeitos; 2 - dispositivo com dois defeitos; 3 - dispositivo com um defeito, e 4 - dispositivo sem nenhum defeito (estado de novo).

TABELA 1. Principais defeitos verificados nos itens avaliados.

\begin{tabular}{ll}
\hline \multicolumn{1}{c}{ Item Avaliado } & \multicolumn{1}{c}{ Principais Defeitos ${ }^{1}$} \\
\hline Toldo solar & $\begin{array}{l}\text { Amassado; rachaduras; cantos vivos; faltando uma } \\
\text { parte (2 defeitos); ferrugem. }\end{array}$ \\
\hline $\begin{array}{l}\text { Proteção das partes ativas (TDP e pás do } \\
\text { ventilador) }\end{array}$ & $\begin{array}{l}\text { Amassada; rachada; solta; faltando alguma parte; com } \\
\text { cantos vivos; com alguma parte quebrada. }\end{array}$ \\
\hline Degraus de acesso & $\begin{array}{l}\text { Tortos ou amassados; com rachaduras; uma parte } \\
\text { quebrada (2 defeitos); com pontas vivas. }\end{array}$ \\
\hline Apoio para os braços & Quebrado; rachado; um deles ausente; solto. \\
\hline Estofamento do banco do operador & $\begin{array}{l}\text { Rasgos; sem cobertura (2 defeitos); com espuma } \\
\text { aparecendo; furos; faltando espuma. }\end{array}$ \\
\hline Isolamento térmico da transmissão & Rasgado; furado; faltando alguma parte (2 defeitos). \\
\hline Alavancas de câmbio & $\begin{array}{l}\text { Ausência da parte superior; alavanca quebrada (2 } \\
\text { defeitos); presença de pontas vivas. }\end{array}$ \\
\hline Cinto de segurança & $\begin{array}{l}\text { Presença de impurezas no mecanismo de engate (2 } \\
\text { defeitos); rasgado. }\end{array}$ \\
\hline Sistema de iluminação (todos os dispositivos) & $\begin{array}{l}\text { Um não liga (2 defeitos); um sem cobertura; um com a } \\
\text { cobertura rachada. }\end{array}$ \\
\hline${ }^{1}$ Quando os problemas indicados valem mais de um defeito, o número é apresentado entre parênteses.
\end{tabular}

O próximo passo no cálculo do CPS foi ponderar a pontuação de cada um desses itens, em função de sua importância em termos de segurança. Da mesma forma, os fatores de correção foram estipulados de forma que a pontuação máxima do espécime avaliado fosse igual a 100 . Isso foi conseguido por meio do emprego da eq.(3).

$$
\mathrm{P}_{\mathrm{f}}=\mathrm{Pf}
$$

em que,

$\mathrm{P}_{\mathrm{f}}$ - pontuação final do item;

$\mathrm{P}$ - pontuação anterior do item, podendo ser a pontuação corrigida $\left(\mathrm{P}_{c}\right)$ se o estado de conservação do mesmo foi analisado, e

f - fator de correção (Tabela 2), definido subjetivamente, utilizando como base referências bibliográficas.

Finalmente, o valor do CPS é dado pela soma da pontuação final obtida por cada um dos itens especificados anteriormente. Verifica-se que a soma dos fatores de correção listados na Tabela 2 corresponde a 10. Assim, um trator que receba nota $10 \mathrm{em}$ todos os itens avaliados obterá, segundo a eq.(3), uma pontuação total igual a 100. 
TABELA 2. Fatores de correção empregados para o cálculo da pontuação final de cada item referente ao CPS.

\begin{tabular}{lclc}
\hline \multicolumn{1}{c}{ Item } & $\mathrm{f}$ & \multicolumn{1}{c}{ Item } & $\mathrm{f}$ \\
\hline Estrutura de proteção contra o capotamento & 1,13 & $\begin{array}{l}\text { Controle do sistema hidráulico de três pontos } \\
\text { fora do posto de operação }\end{array}$ & 0,34 \\
Proteção das pás do ventilador & 0,11 & Cor vermelha do comando da TDP & 0,06 \\
Protecão da TDP & 0,39 & Rapidez no desligamento da TDP & 0,14 \\
Invólucro adicional TDP & 0,06 & Acelerador de pé & 0,11 \\
Advertência de risco de capotamento & 0,06 & Cinto de segurança & 0,68 \\
Advertência de risco da TDP & 0,06 & Luz de freio & 0,34 \\
Orientações sobre a partida do motor & 0,06 & Luz de ré & 0,11 \\
Orientações sobre o acoplamento implementos & 0,06 & Indicadores de direção & 0,34 \\
Existência degraus de acesso e saída & 0,14 & Luzes de advertência & 0,11 \\
Adequação da altura do primeiro degrau & 0,06 & Faróis dianteiros & 0,56 \\
Adequação da largura dos degraus & 0,06 & Lanternas traseiras & 0,56 \\
Adequãão da distância entre os degraus & 0,06 & Farol de trabalho & 0,11 \\
Degraus com superfície antiderrapante & 0,11 & Encosto do assento & 0,11 \\
Batente vertical de ambos os lados dos degraus & 0,06 & Dispositivo que une os pedais dos freios & 0,23 \\
Inclinação da escada & 0,08 & Buzina & 0,23 \\
Corrimão e/ou manípulos & 0,23 & Espelho retrovisor & 0,34 \\
Tipo de posto de operação & 0,28 & Contrapesos dianteiros & 0,28 \\
Proteção da tubulação de escape & 0,28 & Pneus & 0,17 \\
Superfície antiderrapante no posto de operação & 0,06 & Engate rápido no sistema hidráulico de levante & 0,45 \\
Superfície antiderrapante nos pedais & 0,11 & Assento adicional & 0,56 \\
Dispositivo contra partida acidental & 0,34 & Dispositivo de drenagem de água no posto de & 0,14 \\
Segurança nos comandos & 0,23 & operação & \\
\hline
\end{tabular}

O procedimento geral para o cálculo do coeficiente parcial relativo à ergonomia (CPE) é semelhante ao apresentado anteriormente para o CPS. Modificam-se apenas os itens avaliados, bem como a pontuação e os fatores de correção utilizados para a ponderação dos valores. A pontuação atribuída a cada item, de acordo com a existência e o tipo do mesmo, é abaixo apresentada:

- Presença de regulagem horizontal, regulagem vertical, regulagem de pressão e regulagem de inclinação do assento, regulagem de inclinação do encosto, estofamento do assento, regulagem do volante de direção, isolamento térmico da transmissão, isolamento térmico do motor: não (0 ponto); $\operatorname{sim}(10$ pontos);

- Presença de cabina ou toldo: ausentes ( 0 ponto); toldo (2,5 pontos); cabina (10 pontos); pontos);

- Apoio para os braços: ausente (0 ponto); sim, sem regulagem (7 pontos); com regulagem (10

- Posição das alavancas de câmbio: central (3 pontos); lateral (10 pontos), e

- Direcionamento dos gases de escapamento: abaixo do nível do toldo solar/cabina (0 ponto); acima do toldo solar/cabina ou não aplicável - caso em que o trator não possua nem cabina nem toldo: 10 pontos.

Posteriormente, os itens em que a análise do estado de conservação foi executada (Tabela 1), tiveram a sua pontuação corrigida segundo a nota atribuída durante a referida avaliação, tal como executado para o CPS. Da mesma forma, todos os itens tiveram a sua pontuação ponderada em razão da importância relativa dos mesmos, empregando a mesma fórmula utilizada para o CPS. Os valores dos diferentes fatores de correção são apresentados na Tabela 3. O valor do coeficiente parcial de 
ergonomia (CPE), de maneira análoga ao CPS, foi obtido por meio da soma das pontuações finais referentes a cada item relativo à ergonomia.

TABELA 3. Fatores de correção empregados para o cálculo da pontuação final de cada item referente ao CPE.

\begin{tabular}{lclc}
\hline \multicolumn{1}{c}{ Itens } & $\mathrm{f}$ & \multicolumn{1}{c}{ Itens } & $\mathrm{f}$ \\
\hline Presença de cabina ou toldo solar & 3,90 & Presença e regulagem de apoio braços & 0,26 \\
Regulagem horizontal do assento do operador & 0,52 & Estofamento do assento do operador & 0,65 \\
Regulagem vertical do assento do operador & 0,39 & Regulagem do volante de direção & 0,52 \\
Regulagem de pressão do assento do operador & 0,52 & Isolamento térmico da transmissão & 0,78 \\
Regulagem de inclinação do assento & 0,26 & Isolamento térmico do motor & 0,78 \\
Regulagem de inclinação do encosto & 0,26 & Direcionamento dos gases de escape & 0,38 \\
Posição das alavancas de câmbio & 0,78 & & \\
\hline
\end{tabular}

O CPCG englobou todos os fatores que influenciam tanto na ergonomia quanto na segurança das referidas máquinas. A metodologia de cálculo foi semelhante à já apresentada anteriormente para os outros dois coeficientes. A pontuação referente a cada item englobado pelo CPCG foi a seguinte:

- Tempo de uso, calculado em função do ano de fabricação: mais de 30 anos (0 ponto); de 21 a 30 anos (1,5 ponto); de 11 a 20 anos (5 pontos); de 6 a 10 anos (7,5 pontos); até 5 anos (10 pontos);

- Estado geral de manutenção: ruim (0 ponto); médio (5 pontos); bom (10 pontos).

Posteriormente, a pontuação atribuída segundo esses critérios foi ponderada segundo um fator de correção f. Para o tempo de uso, o valor $\mathrm{f}$ empregado foi igual a 6 , enquanto para o estado de manutenção geral o valor de f foi 4 . Somando-se a pontuação corrigida obtida pelos tratores agrícolas nesses dois itens, chegou-se ao valor do CPCG.

O cálculo do coeficiente parcial de ergonomia e segurança em tratores agrícolas foi efetuado por meio da ponderação dos valores obtidos para o CPS, CPE e CPCG. A equação empregada foi a seguinte:

$$
\text { COPES }=(0,7 \mathrm{CPS})+(0,22 \mathrm{CPE})+(0,08 \mathrm{CPCG})
$$

Para a análise estatística dos dados relativos a freqüências, foi empregado o teste nãoparamétrico do qui-quadrado a $1 \%$ de probabilidade de erro (SIEGEL, 1956), tendo em vista a inadequação de dados dessa natureza às pressuposições dos modelos dos testes paramétricos tradicionais. Já o teste de Wilcoxon foi usado para comparar valores médios oriundos de amostras diferentes. Para a análise de correlação, a significância, bem como o valor do coeficiente foram determinados pelo teste de Spearman, a 1\% de probabilidade de erro (SIEGEL, 1956). Já a equação de regressão foi ajustada usando o método dos mínimos quadrados por meio do uso do programa computacional Microsoft Excel ${ }^{\circledR}$.

\section{RESULTADOS E DISCUSSÃO}

Na Tabela 4, são expostos os valores do intervalo de variação e da média geral dos valores do COPES e seus componentes. Para todos os coeficientes, houve uma grande variação entre os tratores amostrados. Além disso, a média geral para o COPES e os principais componentes (CPS e CPE) foi inferior a 35, o que pode ser considerado um valor bastante baixo levando-se em consideração os coeficientes máximos obtidos, que ficaram acima de 80 . Ainda, com relação à Tabela 4 , foi aplicado o teste de Wilcoxon, a 1\% de probabilidade de erro, para comparar os valores médios do CPS e CPE entre si. Obteve-se que os tratores agrícolas avaliados têm limitações de magnitude semelhantes tanto para as condições de segurança quanto para as características ergonômicas. 
TABELA 4. Média e amplitude de variação do CPS, CPE, CPCG e COPES.

\begin{tabular}{ccc}
\hline Coeficientes & Intervalo de Variação & Média \\
\hline CPS & $6,23-83,75$ & 33,14 \\
CPE & $5,65-99,22$ & 33,49 \\
CPCG & $0,00-100,00$ & 52,08 \\
COPES & $7,64-84,80$ & 34,73 \\
\hline
\end{tabular}

Na Tabela 5, evidenciam-se as más condições de segurança dos tratores agrícolas, por meio da distribuição do COPES e seus componentes em diferentes classes de valores. Pode-se observar que, para o CPS, CPE e COPES, a maior parte dos tratores amostrados, respectivamente, 69,71; 72,57 e $68,58 \%$ apresentaram valores inferiores a 40 . Por outro lado, considerando-se os mesmos coeficientes citados anteriormente, menos de $3 \%$ dos tratores analisados obtiveram valores entre 80 e 100 . As diferenças entre as freqüências absolutas de cada classe de valores para esses coeficientes foram significativas, conforme o teste do qui-quadrado, a $1 \%$ de probabilidade de erro. O comportamento dos tratores foi um pouco diferente para o CPCG. Para esse coeficiente, o teste do qui-quadrado mostrou que não houve diferenças significativas entre as freqüências absolutas dos tratores inclusos nas diferentes classes de valores.

TABELA 5. Distribuição dos tratores agrícolas usados segundo as classes de valores do CPS, CPE, CPCG e COPES.

\begin{tabular}{crrrrrrrr}
\hline \multirow{2}{*}{ Classes } & \multicolumn{9}{c}{ Coeficientes } \\
\cline { 2 - 9 } & \multicolumn{2}{c}{ CPS } & \multicolumn{1}{c}{ CPE } & \multicolumn{2}{c}{ CPCG } & \multicolumn{2}{c}{ COPES } \\
\cline { 2 - 9 } $\mathrm{N}^{\mathbf{0}}$ & \multicolumn{1}{c}{$\%$} & $\mathrm{~N}^{\mathbf{0}}$ & \multicolumn{1}{c}{$\%$} & $\mathrm{~N}^{\mathbf{0}}$ & \multicolumn{1}{c}{$\%$} & $\mathrm{~N}^{\mathbf{0}}$ & \multicolumn{1}{c}{$\%$} \\
\hline 0 a 20,00 & 58 & 33,14 & 40 & 22,86 & 33 & 18,86 & 46 & 26,29 \\
20,01 a 40,00 & 64 & 36,57 & 87 & 49,71 & 35 & 20,00 & 74 & 42,29 \\
40,01 a 60,00 & 29 & 16,57 & 32 & 18,29 & 37 & 21,14 & 31 & 17,70 \\
60,01 a 80,00 & 20 & 11,43 & 11 & 6,29 & 29 & 16,57 & 20 & 11,43 \\
80,01 a 100,00 & 4 & 2,29 & 5 & 2,85 & 41 & 23,43 & 4 & 2,29 \\
Total & 175 & 100,00 & 175 & 100,00 & 175 & 100,00 & 175 & 100,00 \\
\hline
\end{tabular}

Segundo FEBO \& PESSINA (1995), a presença dos diferentes dispositivos de segurança e ergonomia, na maioria dos casos, tende a diminuir para os tratores mais antigos. O comportamento do COPES em relação ao tempo de uso dos tratores agrícolas expressou o mesmo comportamento, conforme demonstra a Figura 1. Nessa Figura, verifica-se que o COPES decresce em escala logarítmica, conforme o tempo de uso dos tratores analisados aumenta. A equação de regressão encontrada ajustou-se bem aos dados, haja vista que o coeficiente de determinação foi de 0,8018 . Da mesma forma, a análise de correlação pelo método de Spearman indicou que o coeficiente de correlação COPES x tempo de uso foi de $-0,8948$, o qual foi significativo a $1 \%$ de probabilidade de erro. Cabe destacar que esse comportamento do COPES constitui-se em um indicativo de sua aplicabilidade, a partir do momento em que se mostrou sensível para diferenciar de maneira significativa os tratores mais recentes dos mais antigos, no que se refere à qualidade ergonômica e de segurança. 


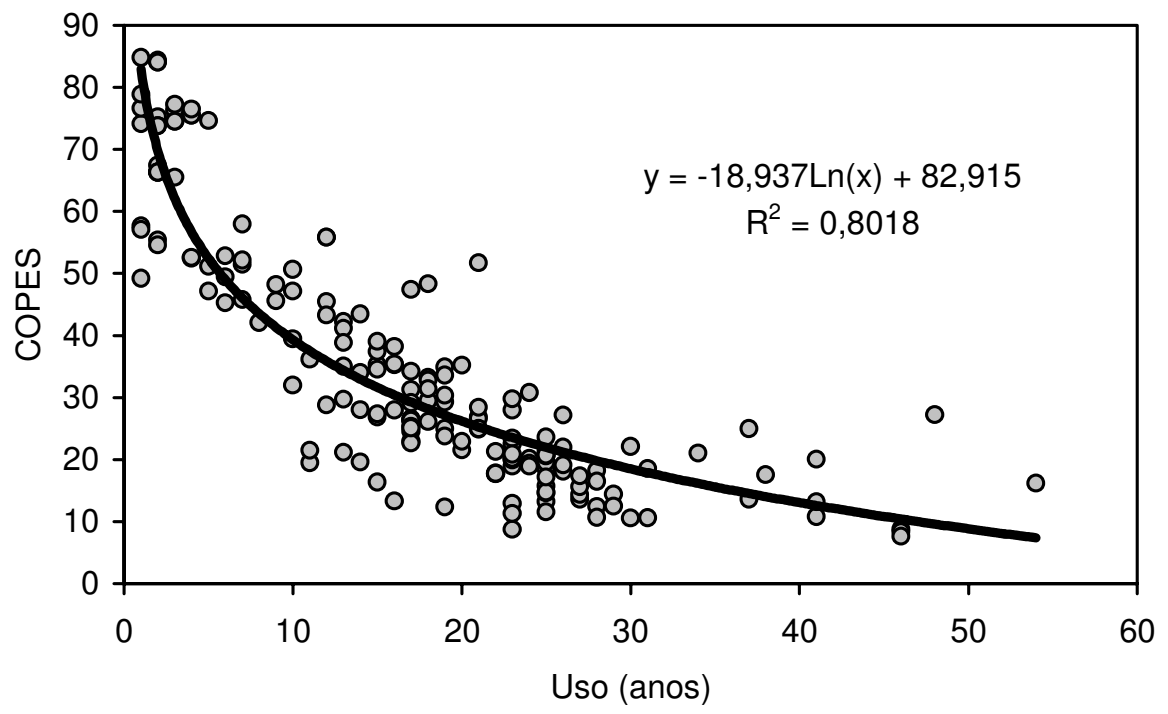

FIGURA 1. Comportamento do coeficiente parcial de ergonomia e segurança em tratores agrícolas (COPES) em função do tempo de uso.

\section{CONCLUSÕES}

O COPES é uma importante ferramenta para a avaliação quantitativa das condições de ergonomia e segurança em tratores agrícolas, apresentando sensibilidade às variações apresentadas pelos tratores a essas características. Da mesma forma, o COPES indicou que os tratores agrícolas usados na região onde foi executada esta pesquisa, apresentam grandes limitações ergonômicas e de segurança, as quais foram mais evidentes para os tratores mais antigos.

\section{REFERÊNCIAS}

ASSOCIAÇÃO BRASILEIRA DE NORMAS TÉCNICAS. Tratores e máquinas agrícolas $e$ florestais: Recursos técnicos para garantir a segurança - Parte 1: Geral. NBR ISO 4254-1. Rio de Janeiro, 1999. $13 \mathrm{p}$.

CARDELLA, B. Segurança no trabalho e prevenção de acidentes. São Paulo: Atlas, 1999. 254 p.

CUTULI, J. A.; CAMPANUCCI, L.; TUSIANI, M.O. Seguridad y higiene en el trabajo. Buenos Aires: Instituto Argentino, 1977. 512 p.

ERIKSON, G. Underlying causes of accidents in agriculture. In: INTERNATIONAL CONFERENCE ON AGRICULTURAL ENGINEERING, 1996, Madrid. Paper...Madrid: CIGR, 1996. (AgEng Paper, 96G-033).

FEBO, P.; PESSINA, D. Survey of the working condition of used tractors in Northern Italy. Journal of Agricultural Engineering Research, London, v.62, n.3, p.193-202, 1995.

MARQUEZ, L. Solo tractor'90. Madrid: Laboreo, 1990. 231 p.

PURCELL, W.F.H. Human factors in tractor design. In: LILJEDAHL, J.B.; TURNQUIST, P.K.; SMITH, D.W.; HOKI, M. Tractors and their power units. 4.ed. St. Joseph: ASAE, 1996. p.203-32.

SCHLOSSER, J.F.; DEBIASI, H. Acidentes com tratores agrícolas: caracterização e prevenção. Santa Maria: UFSM, 2001. 86 p. (Caderno Didático, 8). 
SIEGEL, S. Nonparametric statistics for the behavioral sciences. New York: McGraw-Hill Book Company, 1956. 312 p.

SUUTARINEN, J. Tractor accidents in Finland, 1987. Henlsinki: University of Helsinki, Department of Agricultural Engineering and Household Technology, 1991. 50 p. (Research Bulletin, 62).

ZÓCCHIO, A . Prática da prevenção de acidentes: ABC da segurança do trabalho. 2.ed. São Paulo: Atlas, 1971. 173 p.

WITNEY, B. Operator productivity. In: Choosing and using farm machines. Harlow: Longman Scientific and Technical, 1988. p.28-94.

YADAV, R.; TEWARI, V.K. Tractor operator workplace design - a review. Journal of Terramechanics, Oxford, v.35, n.1, p.41-53, 1998. 